\title{
Group Decision Making with Incomplete Reciprocal Preference Relations Based on Multiplicative Consistency *
}

\author{
Etienne E. Kerre ${ }^{1 \dagger}$, Atiq-ur-Rehman ${ }^{2}$, Samina Ashraf ${ }^{3}$ \\ ${ }^{1}$ Department of Applied Mathematics, \\ Computer Science and Statistics, \\ Ghent university, Belgium \\ E-mail: etienne.kerre@ugent.be \\ ${ }^{2}$ Department of Mathematics, \\ COMSATS Institute of Information Technology, \\ Lahore, Punjab, Pakistan \\ E-mail: atiq.math@gmail.com \\ ${ }^{3}$ Director, Quality Enhancement cell, \\ Lahore College for Women University \\ Lahore, Punjab, Pakistan \\ E-mail: saminaa561@gmail.com
}

Received 12 January 2018

Accepted 8 May 2018

\begin{abstract}
This paper comprises a new iterative method for multi-person decision making based on multiplicative consistency with incomplete reciprocal preference relations (IRPRs). Additionally, multiplicative transitivity property of reciprocal preference relation (RPR) is used at the first level to estimate the unknown preference values and get the complete preference relation, then it is confirmed to be multiplicative consistent by using transitive closure formula. Following this, expert's weights are evaluated by merging consistency and trust weights. The consistency weights against the experts are evaluated through multiplicative consistency investigation of the preferences given by each expert, while trust weights play the role to measure the level of trust for an expert. The consensus process determines whether the selection procedure should start or not. If it results in negative, the feedback mechanism is used to enhance the consensus degree. At the end, a numerical example is given to demonstrate the efficiency and practicality of the proposed method.
\end{abstract}

Keywords: Fuzzy set; Group decision making; Reciprocal preference relation; Incomplete preference relation; Multiplicative consistency.

\footnotetext{
* Group Decision Making with Incomplete Reciprocal Preference Relations Based on Multiplicative Consistency.

$\dagger$ Etienne E. Kerre, Fuzziness and Uncertainty Modeling Research Unit B-9000 Gent, Belgium.

$\ddagger$ etienne.kerre@ugent.be
} 


\section{Introduction}

Decision making (DM) is an intellectual procedure which is used to select the best option(s) amongst several different options, it initiates when we have to do something but do not know what. Every individual faces DM situations in his/her daily life: common examples for these situations are shopping, to choose what to eat, and deciding whom or what to vote for in an election or referendum, and can be categorized in several different groups according to certain characteristics as the source(s) for the information and the preference representation formats that are used to solve the decision problem. In our framework, the selection of the best alternative(s) from a predetermined set $X=\left\{x_{1}, x_{2}, x_{3}, \ldots, x_{n}\right\}, n \geqslant 2$ of possible alternatives is the goal.

DM is not only the case for a single expert, where he/she compares a finite set of alternatives and construct a preference relation, but some decision problems have to be solved by a group of experts who work together to find the best alternative(s) from a set of feasible alternatives. This decision making with multiple experts is called group decision making (GDM) or also known as multi-person decision making. To solve a GDM problem appropriately, two key processes play an essential role:

(i) consensus process;

(ii) selection process.

The prior is an iterative process which is composed of several consensus rounds, where the experts accept to negotiate diverse sentiments to have an acceptable level, but a unanimous or full consensus is often not achievable in practice ${ }^{13}$. After getting the experts' opinions close enough, the selection process takes place which aims to rank and select (a) suitable alternative(s) from a given set of feasible alternatives. When a number of experts interact to reach a decision, each expert may have exclusive inspirations or objectives and a different decision procedure, but has a common interest in approaching to select the best option(s).

In modern era, various consensus models have been proposed in literature for GDM against a number of preference relations ${ }^{2,9,12,25}$. However, there may arise some situations for DM problems in which experts are unable to provide precise and complete assessments due to the pressure to make a quick decision, Complexity, or incomprehensive information against the problem to be explained, such situations result in incomplete fuzzy preference relations (IFPRs) i.e., some preference values are missing. GDM in IFPRs environment has been receiving an intensive interest of researchers, and various procedures have been presented to determine unknown preference values ${ }^{17}$. Such as, a least squared procedure was proposed by Gong ${ }^{8}$ in 2008 to determine the priority vector for GDM in incomplete preference relations' (IPRs) environment. In 2010, a goal programming model was presented by Fan and Zhang $^{7}$ for GDM to deal with IPRs in three formats.In 2013, Xu et al. proposed logarithmic least squares method to evaluate the priority weights in GDM dealing with IFPRs and develop the acceptable fuzzy consistency ratio ${ }^{20}$. Xu and Wang ${ }^{21}$ in 2013, presented eigenvector method to repair an IFPR with consistency test relation. In 2015, Xu et al. ${ }^{22}$ presented a least deviation method to evaluate the priority weights for GDM in IRPRs environment. In 2015, the trust based consensus model and aggregation method for GDM were investigated by $\mathrm{Wu}$ et al. ${ }^{19}$ in the context of incomplete linguistic information.

Moreover, consistency is an important issue to accept when data are provided by the experts 5,10 and is linked to the transitivity property. In 2007, Herrera-Viedma et al. ${ }^{12}$ presented an additive consistency based iterative scheme to evaluate the missing preferences in IFPRs. In 2008, Alonso et al. ${ }^{1}$ extended the idea proposed by Herrera-Viedma et al. ${ }^{12}$ to investigate the missing information against several preference formats. In 2014, a consensus based model was proposed by $\mathrm{Wu}$ and Chiclana ${ }^{18}$ for multiplicative consistency of reciprocal intuitionistic preference relations and discussed its application to estimate the unknown preference degrees. In 2015, a confidence consistency driven approach was proposed by Ureña et al. ${ }^{16}$ to handle GDM problems with incomplete reciprocal intuitionistic preference relations. This approach deals with confidence level and implemented for both consistency 
and confidence in the determination procedure. In 2015, Ashraf et al. ${ }^{3}$ proposed a new method for GDM with IFPRs based on $T$-consistency and the order consistency, where $T$ stands for a triangular norm. In 2017, Xu et al. ${ }^{23}$ developed a consensus based model for hesitant FPRs and used it in water allocation management as an application.

To achieve an acceptable consensus level, feedback mechanism plays an important role in certain consensus measures ${ }^{6,25,26,27}$. In ${ }^{25}$, Zhang et al. developed a consensus building method based on multiplicative consistency for GDM with IRPRs. Inspired by the work of Zhang et al. ${ }^{25}$, we observed that the multiplicative transitivity property i.e., $\frac{r_{i k}}{r_{k i}}=$ $\frac{r_{i j} r_{j k}}{r_{j i} r_{k j}}$ for all $j \neq i, k \in\{1,2,3,4\}$ is violated or overlooked. For example, if we consider the aggregated group preference matrix constructed in ${ }^{25}$ :

$$
P=\left[\begin{array}{cccc}
0.5 & 0.43 & 0.58 & 0.74 \\
0.57 & 0.5 & 0.75 & 0.83 \\
0.42 & 0.25 & 0.5 & 0.74 \\
0.26 & 0.17 & 0.26 & 0.5
\end{array}\right]
$$

the multiplicative transitivity for, say, $i=1$ and $k=2$ implies that $\frac{r_{12}}{r_{21}}=\frac{r_{1 j} r_{j 2}}{r_{j 1} r_{2 j}}$ must be satisfied for $j=3,4$, but from matrix $P$ we have: $\frac{r_{12}}{r_{21}}=0.7543859649$ and $\frac{r_{13} r_{32}}{r_{31} r_{23}}=0.4603174603, \frac{r_{14} r_{42}}{r_{41} r_{24}}=0.5829471733$, we can see that the aggregated matrix constructed by Zhang et al. ${ }^{25}$ does not satisfy the multiplicative consistency. We believe that the multiplicative transitivity property is very hard to satisfy while decimal numbers are being used but to ensure consistency the error can be minimized upto an insignificant level.

In this paper, we present an improved method for consensus building in group decision making based on multiplicative consistency with IRPRs. At the first step, we evaluate the missing preferences of IRPRs based on the multiplicative transitivity. Then, we construct the modified consistency matrices of experts which satisfy the multiplicative consistency and measure the level of consistency. The degrees of importance are assigned to experts based on consistency weights aggregated with trust weights. The proposed method provides us with a valuable way for consensus building in group decision making based on multiplicative consistency with incomplete preference relations.
The rest of the paper is organized as follows. In Section 2, we focus on some preliminaries used in this paper. In Section 3, we present a new procedure to estimate the missing preferences in incomplete preference relations based on the multiplicative transitivity and construct the modified consistency matrices which satisfy the multiplicative consistency. In Section 4, the proposed GDM process is detailed. In Section 5, an example is given to illustrate the realism and achievability of the proposed technique. Last section includes some conclusions.

\section{Preliminaries}

In 1965, Zadeh introduced fuzzy set theory ${ }^{24}$, designated with a number between 0 and 1 , to cope with imprecise and uncertain information working in complex situation.

Definition 1. Fuzzy Set ${ }^{24}$ : A fuzzy set $A$ on the universe of discourse $X$ is a mapping from $X$ to $[0,1]$. and is denoted by $A=\{(x, A(x))\}$. For any $x \in X$, the value $A(x)$ is called the degree of membership of $x$ in $A$ i.e., $A(x)=\operatorname{Degree}(x \in A)$, and the map $A: X \rightarrow[0,1]$ is called a membership function.

Definition 2. Reciprocal Preference Relation $(\mathrm{RPR})^{5}$ : An RPR $R$ on a set $X$ of alternatives $X=\left\{x_{1}, x_{2}, \ldots, x_{n}\right\}$ is characterized by a membership function $R\left(x_{i}, x_{j}\right)=r_{i j}$, satisfying $r_{i j}+r_{j i}=1$ for all $i, j \in\{1,2,3, . ., n\}$.

An RPR may be conveniently denoted by matrix $R=\left(r_{i j}\right)_{n \times n}$, with the following interpretations:

$$
R=\left(r_{i j}\right)_{n \times n}=\left[\begin{array}{ccccc}
0.5 & r_{12} & . & \cdot & r_{1 n} \\
r_{21} & 0.5 & . & \cdot & r_{2 n} \\
\cdot & \cdot & \cdot & & \cdot \\
\cdot & \cdot & & \cdot & \cdot \\
r_{n 1} & r_{n 2} & . & . & 0.5
\end{array}\right]
$$

where $r_{i j}$ represents the preference value of alternative $i$ over alternative $j$, and all $r_{i i}=0.5$. If $r_{i j}=0.5$, this indicates that there is no difference between the two alternatives; if $r_{i j}>0.5$, it implies that alternative $i$ is superior to alternative $j$.

Definition 3. Incomplete Reciprocal Preference Relation $(I R P R)^{11}$ : An IRPR $R=\left(r_{i j}\right)_{n \times n}$ carries at least one unknown preference value $r_{i j}$ for which the 
expert does not have a clear idea of the degree of preference of alternative $x_{i}$ over the alternative $x_{j}$.

Definition 4. Multiplicative Transitivity Property ${ }^{15}$ : An RPR $R=\left(r_{i j}\right)_{n \times n}$ on a finite set $X$ of alternatives is said to be multiplicative transitive if and only if

$$
\frac{r_{i j}}{r_{j i}}=\frac{r_{i k} r_{k j}}{r_{j k} r_{k i}}
$$

holds for all $i, j, k \in\{1,2,3, \ldots, n\}$ with non-zero preference values. The multiplicative transitivity leads to multiplicative consistency of RPR.

\section{Evaluating missing preferences}

This section puts forward a new scheme to estimate the unknown preference values in an IRPR based on multiplicative transitivity. Moreover, the proposed procedure is used to construct a multiplicative consistent matrix.

So as to determine missing preferences in an IRPR $R=\left(r_{i j}\right)_{n \times n}$, the pairs of alternatives for known and unknown preferences are signified in form of following sets:

$$
\begin{aligned}
& K_{v}=\left\{(i, j) \mid r_{i j} \text { is known }\right\} ; \\
& U_{v}=\left\{(i, j) \mid r_{i j} \text { is unknown }\right\},
\end{aligned}
$$

where $K_{v}$ is the set of pairs of alternatives with known preference degrees whereas $U_{v}$ represents the set of pairs of alternatives with missing preference degrees. The preference value of alternative $x_{i}$ over $x_{j}$ belongs to $[0,1]$ (i.e., $r_{i j} \in[0,1]$ ). Since $r_{i j}+r_{j i}=1$ for $1 \leqslant i \leqslant n$ and $1 \leqslant j \leqslant n$, therefore, multiplicative transitivity (3) can be written as:

$$
r_{i j}=\frac{r_{i k} \cdot r_{k j}}{1-r_{i k}-r_{k j}+2 r_{i k} \cdot r_{k j}}
$$

where $1>r_{i j}>0 \forall i, j \in\{1,2,3, \ldots, n\}$. Hence, we can define following set of intermediate alternative $x_{j}$ which can be used to determine the unknown preference value $r_{i k}$ of alternative $x_{i}$ over alternative $x_{k}$ :

$W_{i j}=\left\{k \neq i, j \mid(i, k) \in K_{v},(k, j) \in K_{v}\right.$ and $\left.(i, j) \in U_{v}\right\}$,

for $1 \leqslant i \leqslant n, 1 \leqslant j \leqslant n$ and $1 \leqslant k \leqslant n$. As consistency is an essential property for RPR, expression
(6) can be used to estimate the missing preference value by using other preference values to maintain the internal consistency of RPR. Definitely, the unknown preference value $r_{i j}(i \neq j)$ can be calculated using an intermediate alternative $x_{k}(k \neq i, j)$ based on (6), but the aggregated value (global value) of $r_{i j}$ is obtained by using the max aggregation operator and is the degree of preference of alternative $x_{i}$ over the alternative $x_{j}$ :

$$
r_{i j}= \begin{cases}\max _{k \in W_{i j}}\left(\frac{r_{i k} \cdot r_{k j}}{1-r_{i k}-r_{k j}+2 r_{i k} \cdot r_{k j}}\right), & \text { if }\left|W_{i j}\right| \neq 0 \\ 0.5, & \text { otherwise }\end{cases}
$$

where $\left|W_{i j}\right|$ is the cardinality of the set $W_{i j}$. Aggregation and fusion of information are basic concerns for all kinds of knowledge based systems, from image processing to decision making, from pattern recognition to machine learning. From a general point of view we can say that aggregation has for purpose the simultaneous use of different pieces of information (provided by several sources) in order to come to a conclusion or a decision. Here max aggregation operator is used for optimal attitude while other operators as min and average etc can also be used. The value $r_{j i}$ is estimated by using reciprocity after having the value of $r_{i j}$ as:

$$
r_{j i}=1-r_{i j}
$$

New sets of the pairs of alternatives for known and unknown preference values are evaluated as follows:

$$
\begin{aligned}
& K_{v}^{\prime}=K_{v} \cup\{(i, j)\}, \\
& U_{v}^{\prime}=U_{v}-\{(i, j)\} .
\end{aligned}
$$

After having a complete RPR, it needs to be fully multiplicative consistent RPR $\widetilde{R}=\left(\widetilde{r}_{i j}\right)_{n \times n}$ which can be obtained by calculating $\widetilde{r}_{i j}$ as:

$$
\widetilde{r}_{i j}=\max _{k \neq i, j}\left(r_{i j}, \frac{r_{i k} \cdot r_{k j}}{1-r_{i k}-r_{k j}+2 r_{i k} \cdot r_{k j}}\right)
$$

such that $\widetilde{R}$ is stable with $\widetilde{r}_{i j}+\widetilde{r}_{j i}=1$. It remains in work until two consecutive outcomes result in same RPRs regarding their preferences, at this stage relation $\widetilde{R}$ will satisfy the multiplicative consistency property. 


\section{Iterative procedure for GDM}

Now we turn towards our major task to develop a GDM procedure in IRPRs environment. In this section, a new step-by-step algorithm is presented for GDM based on multiplicative consistency. An explanatory example is given to validate the technique. For ease, the structure of the resolution process is also shown in Figure 1.

Suppose that there are $n$ alternatives $x_{1}, x_{2}, \ldots, x_{n}$ and $m$ experts $E_{1}, E_{2}, \ldots, E_{m}$. Let $R^{q}$ be the fuzzy preference relation for the expert $E_{q}$ shown as follows:

$$
R^{q}=\left(r_{i j}^{q}\right)_{n \times n}=\left[\begin{array}{ccccc}
0.5 & r_{12}^{q} & \cdot & \cdot & r_{1 n}^{q} \\
r_{21}^{q} & 0.5 & \cdot & \cdot & r_{2 n}^{q} \\
\cdot & \cdot & \cdot & \cdot \\
\cdot & \cdot & & \cdot & \cdot \\
r_{n 1}^{q} & r_{n 2}^{q} & \cdot & \cdot & 0.5
\end{array}\right],
$$

where $r_{i j}^{q} \in[0,1]$ is the preference value given by expert $E_{q}$ for alternative $x_{i}$ over $x_{j}, r_{i j}^{q}+r_{j i}^{q}=1,1 \leqslant i \leqslant$ $n, 1 \leqslant j \leqslant n$ and $1 \leqslant q \leqslant m$ and $r_{i i}^{q}=0.5$, for all $i \in$ $\{1,2, \ldots, n\}$ as an alternative cannot be preferred on itself. Some of the preference matrices given by the experts may be IRPRs, because of time constraint or lack of information and complexity.The proposed GDM technique consists of several stages which are described as follows:

\subsection{Estimating missing preferences}

To determine the missing preference values of an IRPR $R^{q}$ given by the expert $E_{q}$, initially, the sets $K^{q}$ and $U^{q}$ of pairs of alternatives for known and unknown preferences are introduced as in (4) and (5) respectively. After this, the multiplicative transitivity based preference values are estimated by using (7)-(11) to construct the complete RPR $R^{q}$.

\subsection{Consistency measures}

After evaluating the complete RPRs, their parallel multiplicative consistent RPRs $\widetilde{R}^{q}$, for $q=$ $1,2,3, \ldots, m$, can also be obtained with the help of (12). We can then approximate the degree of consistency of an RPR $R^{q}$ based on its similarity with the corresponding multiplicative transitivity based $\widetilde{R}^{q}$ by computing their distances ${ }^{25}$.

1. Multiplicative consistency index $(M C I)$ of a pair of alternatives is determined by using:

$$
\operatorname{MCI}\left(r_{i j}^{q}\right)=1-d\left(r_{i j}^{q}, \widetilde{r}_{i j}^{q}\right)
$$

where $d\left(r_{i j}^{q}, \widetilde{r}_{i j}^{q}\right)$ is the error (distance) measured by $\varepsilon r_{i j}^{q}=d\left(r_{i j}^{q}, \widetilde{r}_{i j}^{q}\right)=\left|r_{i j}^{q}-\widetilde{r}_{i j}^{q}\right|$. When $\operatorname{MCI}\left(r_{i j}^{q}\right)=1$ this implies that $\varepsilon r_{i j}^{q}=0$ and there is no inconsistency at all. Apparently, the higher the value of $\operatorname{MCI}\left(r_{i j}^{q}\right)$, the more consistent $r_{i j}^{q}$ is with respect to the rest of the preference values involving alternatives $x_{i}$ and $x_{j}$.

2. MCI associated to a particular alternative $x_{i}$, $1 \leqslant i \leqslant n$, of an RPR is evaluated by:

$\operatorname{MCI}\left(x_{i}\right)=\frac{1}{2(n-1)} \sum_{j=1}^{n}\left(\operatorname{MCI}\left(r_{i j}^{q}\right)+\operatorname{MCI}\left(r_{j i}^{q}\right)\right)$

with $\operatorname{MCI}\left(x_{i}\right) \in[0,1]$. When $\operatorname{MCI}\left(x_{i}\right)=1$ all the preferences involving the alternative $x_{i}$ are fully consistent, otherwise, the lower $\operatorname{MCI}\left(x_{i}\right)$ the more inconsistent these preference values are.

3. Finally we determine $M C I$ of an RPR $R^{q}$ by taking the average of all $M C I$ of alternatives $x_{i}$ :

$$
\operatorname{MCI}\left(R^{q}\right)=\frac{1}{n} \sum_{i=1}^{n} \operatorname{MCI}\left(x_{i}\right)
$$

with $\operatorname{MCI}\left(R^{q}\right) \in[0,1]$. When $\operatorname{MCI}\left(R^{q}\right)=1$ the preference relation $R^{q}$ is fully consistent, otherwise, the lower $\operatorname{MCI}\left(R^{q}\right)$ the more inconsistent $R^{q}$ is.

As soon as the $M C I$ is computed in three levels using expressions (13)-(15), it is rational to assign the higher weights to the experts against the preference relations with larger consistency degrees respectively. Hence, consistency weights can be assigned to the experts by using the relation:

$$
C w\left(E_{q}\right)=\frac{\operatorname{MCI}\left(R^{q}\right)}{\sum_{q=1}^{m} \operatorname{MCI}\left(R^{q}\right)}
$$


where $m$ represents the number of experts, and $\operatorname{MCI}\left(R^{q}\right)$ is the $M C I$ of the opinion of $E_{q}$.

\section{3. $\quad$ Allocating weights to experts}

The trust weight $t w\left(E_{q}\right)$ is the degree of trust on each expert by others such that $\sum_{q=1}^{m} t w\left(E_{q}\right)=m$. Convincingly, the experts with larger trust weights can have higher weight in the aggregation process. Primarily, the same trust weight $t w\left(E_{q}\right)$ has been assigned to each expert. But the trust weights of experts do not remain the same in each consensus round, the procedure given by Zhang et al. ${ }^{25}$ will be used to evaluate the trust weights. Final weights to experts are allocated by emerging respective trust weights and consistency weights under the use of following relation $^{25}$ :

$$
w\left(E_{q}\right)=\frac{t w\left(E_{q}\right) \times C w\left(E_{q}\right)}{\sum_{q=1}^{m} t w\left(E_{q}\right) \times C w\left(E_{q}\right)}
$$

with $\sum_{q=1}^{m} w\left(E_{q}\right)=1$.

\subsection{Consensus measures}

After having the RPRs with complete information, it is necessary to measure the consensus among the experts. Regarding this, similarity matrices $S^{q r}=\left(s_{i j}^{q r}\right)_{n \times n}$ for every pair of experts $\left(E_{q}, E_{r}\right)(q=$ $1,2, \ldots, m-1 ; r=q+1, \ldots, m)$ are to be determined and defined as:

$$
s_{i j}^{q r}=1-d\left(r_{i j}^{q}, r_{i j}^{r}\right)
$$

where $d\left(r_{i j}^{q}, r_{i j}^{r}\right)=\left|r_{i j}^{q}-r_{i j}^{r}\right|$. Then the collective similarity matrix $S=\left(s_{i j}\right)_{n \times n}$ is constructed after aggregating all the similarity matrices by using following relation.

$$
s_{i j}=\frac{2}{m(m-1)} \sum_{q=1}^{m-1} \sum_{r=q+1}^{m} s_{i j}^{q r}
$$

Three different levels are involved to compute the degree of consensus amongst the experts as follows ${ }^{25}$ :
1. At first level, the consensus degree on a pair of alternative $\left(x_{i}, x_{j}\right)$, denoted by $\operatorname{cod}_{i j}$ is defined to estimate the degree of consensus amongst all experts on that pair of alternatives:

$$
\operatorname{cod}_{i j}=s_{i j}
$$

2. At second level, the consensus degree on alternative $x_{i}$, denoted by $C o D_{i}$ is defined to determine the consensus degree amongst all the experts on that alternative:

$$
C o D_{i}=\frac{1}{2(n-1)} \sum_{j=1, j \neq i}^{n}\left(s_{i j}+s_{j i}\right)
$$

3. At third level, the consensus degree on the relation, denoted by $C o R$ is defined to calculate the global degree of consensus amongst all the experts judgments:

$$
\operatorname{CoR}=\frac{1}{n} \sum_{i=1}^{n} \operatorname{CoD}_{i}
$$

Once the global consensus level among all the experts is reached, it requires to compare with a threshold consensus degree $\eta$, generally settled in advance depending upon the nature of problem. If $C o R \geqslant \eta$, this shows that an acceptable level of consensus has been obtained, and the decision process begins. Otherwise, the consensus degree is not stable, and feedback mechanism is originated.

\subsection{Feedback mechanism}

The central aim of feedback mechanism is to provide comprehensive knowledge to experts, so as to change their opinions acceptably to enhance the consensus degree. When consensus is not sufficiently high, then we have to identify the preference values that are to be changed, and following formula helps us in this regard:

$$
R^{q}=\left\{(i, j) \mid \operatorname{cod}_{i j}<\operatorname{CoR} \text { and } r_{i j}^{q} \text { is known }\right\}
$$

for $i, j \in\{1, \ldots, n\}$. The system recommends that the corresponding expert has to increase value if it is smaller than the mean value of the valuations of the 
rest of experts, or decrease it if it is greater than the mean ${ }^{25}$. All at once, there may be a possibility that the minority opinion is precise ${ }^{4}$, a conflict resolution method has been proposed to handle such a situation which contains correct opinions against few experts ${ }^{14}$. We need to find out all alternatives $x_{i}$ whose consensus measures $C o D_{i}$ are lower than $C o R$, and then elaborate the particular pair of experts whose opinions contradict one another on alternative $x_{i}$ to the greatest extent, ( i.e., with the largest deviation of $S$ on alternatives $x_{i}$, which can be measured by the sum of $\left.\sum_{j=1, j \neq i}^{n}\left(s_{i j}+s_{j i}\right)\right)$. With this, we can make them change their initial preferences and, at the same time, accelerate the consensus process.

\subsection{Accumulation phase}

It may quite frequently happen that the preference degree set forth by each expert is weighted differently. As soon as the weights for the experts are estimated, their preferences need to be accumulated into a global one. Determine the collective matrix $R^{C}$ against all experts, shown as follows:

$$
R^{C}=\left(r_{i j}^{c}\right)_{n \times n}=\left(\sum_{q=1}^{m} w\left(E_{q}\right) \times \widetilde{r}_{i j}^{q}\right)_{n \times n}
$$

where $1 \leqslant i \leqslant n, 1 \leqslant j \leqslant n$.

\subsection{Selection phase}

After reaching a satisfactory consensus degree amongst all experts, the selection process is initiated to rank all the alternatives in order to select the best option. For a consistent RPR $\widetilde{R}=\left(\widetilde{r}_{i j}\right)_{n \times n}$, the ranking value $R v\left(x_{i}\right)$ of alternative $x_{i}, i=1, . ., n$, is defined by:

$$
R v\left(x_{i}\right)=\frac{2}{n(n-1)} \sum_{j=1, j \neq i}^{n} \widetilde{r}_{i j}, i=1, . ., n
$$

with $\sum_{i=1}^{n} R v\left(x_{i}\right)=1$

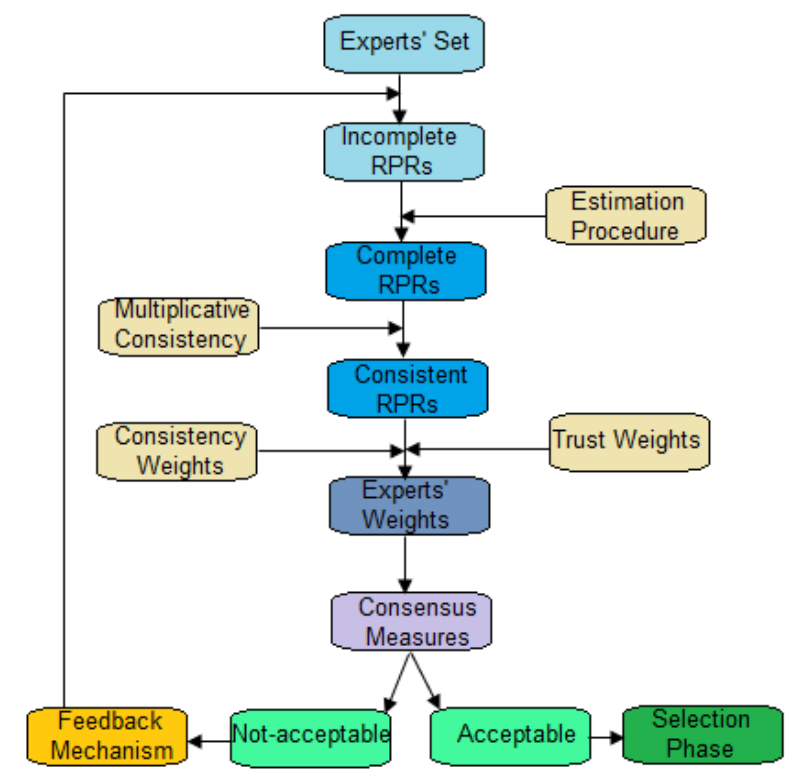

Fig. 1. Resolution process for GDM with IFPRs

\section{Numerical example}

This section deals with a numerical example taken from ${ }^{25}$ in order to demonstrate the process of the proposed method and its effectiveness.

Consider that four experts $E_{1}, E_{2}, E_{3}$ and $E_{4}$ from different fields are requested to select the best alternative out of four alternatives $x_{1}, x_{2}, x_{3}, x_{4}$. The four experts give their RPRs as follows:

$$
\begin{gathered}
R^{1}=\left[\begin{array}{llll}
0.5 & 0.6 & r_{13}^{1} & r_{14}^{1} \\
0.4 & 0.5 & 0.7 & r_{24}^{1} \\
r_{31}^{1} & 0.3 & 0.5 & 0.9 \\
r_{41}^{1} & r_{42}^{1} & 0.1 & 0.5
\end{array}\right], R^{2}=\left[\begin{array}{cccc}
0.5 & 0.6 & 0.7 & r_{14}^{2} \\
0.4 & 0.5 & r_{23}^{2} & 0.7 \\
0.3 & r_{32}^{2} & 0.5 & r_{34}^{2} \\
r_{41}^{2} & 0.3 & r_{43}^{2} & 0.5
\end{array}\right], \\
R^{3}=\left[\begin{array}{cccc}
0.5 & 0.3 & 0.5 & 0.75 \\
0.7 & 0.5 & 0.8 & 0.6 \\
0.5 & 0.2 & 0.5 & 0.8 \\
0.25 & 0.4 & 0.2 & 0.5
\end{array}\right], R^{4}=\left[\begin{array}{cccc}
0.5 & 0.4 & 0.55 & 0.65 \\
0.6 & 0.5 & 0.8 & 0.75 \\
0.45 & 0.2 & 0.5 & 0.7 \\
0.35 & 0.25 & 0.3 & 0.5
\end{array}\right] .
\end{gathered}
$$

The threshold consensus level $\eta$ settled in advance is 0.80 . Now, we perform the following steps to evaluate the result:

\section{Step-i: Estimating the missing preferences}

Initially, all the missing preference values need to be determined using the multiplicative transitivity property mentioned in Section 1. 
Taking $R^{1}$, for example. The sets of pairs of alternatives for known and unknown preference values are determined as follows:

$$
\begin{aligned}
& K_{v}^{1}=\{(1,2),(2,1),(2,3),(3,2),(3,4),(4,3)\}, \\
& U_{v}^{1}=\{(1,3),(3,1),(1,4),(4,1),(2,4),(4,2)\} .
\end{aligned}
$$

All the missing preference values are calculated under the use of (7)-(11) to complete the given IRPR. Hence, the complete RPR $R^{1}$ against expert $E_{1}$ is obtained as follows:

$$
R^{1}=\left[\begin{array}{llll}
0.5000 & 0.6000 & 0.7778 & 0.9692 \\
0.4000 & 0.5000 & 0.7000 & 0.9545 \\
0.2222 & 0.3000 & 0.5000 & 0.9000 \\
0.0308 & 0.0455 & 0.1000 & 0.5000
\end{array}\right] .
$$

Similarly, the complete form of $R^{2}$ can be obtained and given as:

$$
R^{2}=\left[\begin{array}{llll}
0.5000 & 0.6000 & 0.7000 & 0.7778 \\
0.4000 & 0.5000 & 0.6987 & 0.7000 \\
0.3000 & 0.3913 & 0.5000 & 0.6000 \\
0.2222 & 0.3000 & 0.4000 & 0.5000
\end{array}\right] .
$$

\section{Step-ii: Consistency analysis}

Consistency analysis is being conducted to allocate consistency weights to the experts. For this purpose, all complete RPRs are to be converted into their multiplicative consistent forms by using (12), and are given below:

$$
\begin{aligned}
& \widetilde{R}^{1}=\left[\begin{array}{llll}
0.5000 & 0.6000 & 0.7777 & 0.9692 \\
0.4000 & 0.5000 & 0.6999 & 0.9545 \\
0.2223 & 0.3001 & 0.5000 & 0.9000 \\
0.0308 & 0.0455 & 0.1000 & 0.5000
\end{array}\right], \\
& \widetilde{R}^{2}=\left[\begin{array}{llll}
0.5000 & 0.6000 & 0.7000 & 0.7778 \\
0.4000 & 0.5000 & 0.6987 & 0.7000 \\
0.3000 & 0.3913 & 0.5000 & 0.6000 \\
0.2222 & 0.3000 & 0.4000 & 0.5000
\end{array}\right], \\
& \widetilde{R}^{3}=\left[\begin{array}{llll}
0.5000 & 0.4304 & 0.5994 & 0.7916 \\
0.5696 & 0.5000 & 0.6645 & 0.8341 \\
0.4006 & 0.3355 & 0.5000 & 0.7174 \\
0.2084 & 0.1659 & 0.2826 & 0.5000
\end{array}\right], \\
& \widetilde{R}^{4}=\left[\begin{array}{llll}
0.5000 & 0.3504 & 0.5892 & 0.7375 \\
0.6496 & 0.5000 & 0.7267 & 0.8389 \\
0.4108 & 0.2733 & 0.5000 & 0.6620 \\
0.2625 & 0.1611 & 0.3380 & 0.5000
\end{array}\right] .
\end{aligned}
$$

The significant $M C I$ values of the experts are evaluated using (13)-(15), as:

$$
\begin{array}{r}
\operatorname{MCI}\left(R^{1}\right)=0.9999, \operatorname{MCI}\left(R^{2}\right)=1, \\
\operatorname{MCI}\left(R^{3}\right)=0.8794, \operatorname{MCI}\left(R^{4}\right)=0.9372 .
\end{array}
$$

Finally, the consistency weights to the experts are computed by using (16), as:

$$
\begin{aligned}
& C w\left(E_{1}\right)=0.2620, C w\left(E_{2}\right)=0.2620, \\
& C w\left(E_{3}\right)=0.2304, C w\left(E_{4}\right)=0.2456 .
\end{aligned}
$$

\section{Step-iii: Weights to experts}

Primarily, all experts are assigned the same trust weights: $t w\left(E_{1}\right)=1, t w\left(E_{2}\right)=1, t w\left(E_{3}\right)=1$ and $t w\left(E_{4}\right)=1$. Therefore, the weights of the experts remain the same in the first round as the consistency weights based on (17), as:

$$
\begin{aligned}
& w\left(E_{1}\right)=0.2620, w\left(E_{2}\right)=0.2620, \\
& w\left(E_{3}\right)=0.2304, w\left(E_{4}\right)=0.2456 .
\end{aligned}
$$

\section{Step-iv: Consensus measures}

After getting complete RPRs, a mutual similarity relation is computed by aggregating the different similarity matrices among the experts using (18)-(19). Then, the consensus measures are computed at the three levels using (20)-(22).

$$
\begin{aligned}
& S^{12}=\left[\begin{array}{cccc}
1.0 & 1.0 & 0.9223 & 0.8086 \\
1.0 & 1.0 & 0.9088 & 0.7455 \\
0.9223 & 0.9088 & 1.0 & 0.7000 \\
0.8086 & 0.7455 & 0.7000 & 1.0
\end{array}\right], \\
& S^{13}=\left[\begin{array}{cccc}
1.0 & 0.8304 & 0.8217 & 0.8224 \\
0.8304 & 1.0 & 0.9646 & 0.8796 \\
0.8217 & 0.9646 & 1.0 & 0.8174 \\
0.8224 & 0.8796 & 0.8174 & 1.0
\end{array}\right] \text {, } \\
& S^{14}=\left[\begin{array}{cccc}
1.0 & 0.7504 & 0.8115 & 0.7683 \\
0.7504 & 1.0 & 0.9732 & 0.8844 \\
0.8115 & 0.9732 & 1.0 & 0.7620 \\
0.7683 & 0.8844 & 0.7620 & 1.0
\end{array}\right] \text {, } \\
& S^{23}=\left[\begin{array}{cccc}
1.0 & 0.8304 & 0.8994 & 0.9862 \\
0.8304 & 1.0 & 0.9442 & 0.8659 \\
0.8994 & 0.9442 & 1.0 & 0.8826 \\
0.9862 & 0.8659 & 0.8826 & 1.0
\end{array}\right] \text {, } \\
& S^{24}=\left[\begin{array}{cccc}
1.0 & 0.7504 & 0.9892 & 0.9597 \\
0.7504 & 1.0 & 0.8820 & 0.8611 \\
0.9892 & 0.8820 & 1.0 & 0.9380 \\
0.9597 & 0.8611 & 0.9380 & 1.0
\end{array}\right] \text {, }
\end{aligned}
$$




$$
S^{34}=\left[\begin{array}{cccc}
1.0 & 0.9200 & 0.9898 & 0.9459 \\
0.9200 & 1.0 & 0.9378 & 0.9952 \\
0.9898 & 0.9378 & 1.0 & 0.9446 \\
0.9459 & 0.9952 & 0.9446 & 1.0
\end{array}\right]
$$

1. On pair of alternatives:

$$
C o D=\left[\begin{array}{cccc}
1.0 & 0.8469 & 0.8890 & 0.8819 \\
0.8469 & 1.0 & 0.9351 & 0.8719 \\
0.8890 & 0.9351 & 1.0 & 0.8408 \\
0.8819 & 0.8719 & 0.8408 & 1.0
\end{array}\right]
$$

2. On alternatives:

$$
\begin{aligned}
& C o D_{1}=0.8726, C o D_{2}=0.8846, \\
& C o D_{3}=0.8883, C o D_{4}=0.8649 .
\end{aligned}
$$

\section{On relation:}

$$
\operatorname{CoR}=0.8776
$$

Now, the threshold consensus degree $\eta$ settled in advance is compared with global consensus degree $C o R$ of the relation; $\operatorname{CoR}>\eta$. This indicates that the given consensus degree is acceptable amongst the experts, and we have to enter into accumulation phase.

\section{Step-v: Accumulation phase}

$$
R^{C}=\left[\begin{array}{llll}
0.5000 & 0.4992 & 0.6690 & 0.8370 \\
0.5008 & 0.5000 & 0.6697 & 0.8374 \\
0.3310 & 0.3303 & 0.5000 & 0.7176 \\
0.1630 & 0.1626 & 0.2824 & 0.5000
\end{array}\right]
$$

\section{Step-vi: Selection phase}

The relation $R^{C}$ obtained in (26) is clearly multiplicative consistent, therefore, apply (25) to estimate the ranking value $R_{v}\left(x_{i}\right)$ of alternative $x_{i}, 1 \leqslant i \leqslant 4$ as follows:

$$
\begin{aligned}
& R_{v}\left(x_{1}\right)=0.3342, R_{v}\left(x_{2}\right)=0.3347, \\
& R_{v}\left(x_{3}\right)=0.2298, R_{v}\left(x_{4}\right)=0.1013,
\end{aligned}
$$

where $\sum_{i=1}^{4} R v\left(x_{i}\right)=1$ and $R_{v}\left(x_{2}\right)>R_{v}\left(x_{1}\right)>R_{v}\left(x_{3}\right)>$ $R_{v}\left(x_{4}\right)$, therefore, the ranking order of alternatives $x_{1}, x_{2}, x_{3}$ and $x_{4}$ is: $x_{2}>x_{1}>x_{3}>x_{4}$, and the best option is $x_{2}$.
To validate the effectiveness of our proposed method, we compare the results with Zhang et al. ${ }^{25}$ model which yielded the same results i.e., $x_{2}>x_{1}>$ $x_{3}>x_{4}$ as ours. But the aggregated matrix obtained by our method is multiplicative consistent at least correct to three decimal places which can further be extended i.e., if we take $i=1$ and $k=2$, the preference value in aggregated matrix $R^{C}$ is $r_{12}=0.4992$ and the multiplicative consistency requires the same or closed output to $r_{12}$ by $\frac{r_{1 j} r_{j 2}}{r_{j 1} r_{2 j}} r_{21}$ for $j=3,4$ as:

$$
\begin{aligned}
& \frac{r_{13} r_{32}}{r_{31} r_{23}} r_{21}=0.4992, \\
& \frac{r_{14} r_{42}}{r_{41} r_{24}} r_{21}=0.4993 .
\end{aligned}
$$

which shows that $r_{12} \approx \frac{r_{1 j} r_{j 2}}{r_{j 1} r_{2 j}} r_{21}$. Therefore, this check can be made for the whole matrix.

\section{Conclusion}

In this paper, an improved hybrid consensus method for GDM problems based on incomplete RPRs is proposed. The multiplicative transitive property is used to estimate the missing values and transitive closure formula is used to make the matrices multiplicative consistent. The weights of the experts are obtained from the consistency analysis and a calculation of degree of trust. Rationally, the experts with high level of consistency and substantial trust degree should have to assign large weights, in order carry more importance in the aggregation process. Additionally, a feedback mechanism making advice to experts subject to their trust weights and consistency weights was proposed which can accelerate the execution of a higher consensus degree. After reaching a satisfactory consensus degree amongst all experts, the selection process is initiated to rank all the alternatives in order to select the best option. Some numerical examples were provided to highlight the efficiency and feasibility of the proposed method, and some results in comparison with model proposed by Zhang et al. ${ }^{25}$ were given. The results established the practicability of the method, which can help us to gain a greater insight into the GDM process.

To summarize, some of the major advantages of the proposed technique are as follows: 
(1). In the proposed method, the missing preference values for RPRs were estimated using multiplicative transitivity which is more suitable to attain consistency of RPRs as compared with other consistencybased methods; (2). The trustworthiness of experts weights were improved by combining consistency weights and trust weights, and used to measure the consistency of experts estimations upto acceptable level; (3). The proposed method resulted in highly consistent preference relations as compare to other models ${ }^{25}$.

To the best of our knowledge, there are only few hybrid methods of this kind which have been proposed in literature to deal with GDM problems in incomplete RPRs environment. We think that this method handles GDM problems more efficiently and yields more effective agreements.

In future, we will extend the proposed model for multi-criteria decision making.

\section{Acknowledgment}

The authors would like to thank the anonymous reviewers for their constructive comments that helped us to polish and improve the quality of this paper.

\section{References}

1. S. Alonso, F. Chiclana, F. Herrera, E. Herrera-Viedma, J. Alcala-Fdez and C. Porcel, A consistency-based procedure to estimate missing pairwise preference values, Int. J. Intell. Syst. 23 (1) (2008) 155-175.

2. S. Alonso, E. Herrera-Viedma, F. Chiclana and F. Herrera, A web based consensus support system for group decision making problems and incomplete preferences, Inform. Sci. 180 (23) (2010) 4477-4495.

3. A. Rehman, E. E. Kerre and S. Ashraf, Group decision making by using incomplete fuzzy preference relations based on $T$-consistency and the order consistency, Int. J. Intell. Syst. 30 (2015) 120-143.

4. F. J. Cabrerizo, F. Chiclana, R. AI-Hmouz, A. Morfeq, A. S. Balamash and A. Herrera-Viedma, Fuzzy decision making and consensus: challenges, J. Intell. Fuzzy Syst. 29 (3) (2015) 1109-1118.

5. F. Chiclana, E. Herrera-Viedma, S. Alonso and F. Herrera, Cardinal Consistency of Reciprocal Preference Relations: A Characterization of Multiplicative Transitivity, IEEE Transactions on Fuzzy Syst. 17 (1) (2009) 14-23.
6. Y. Dong and H. Zhang, Multiperson decision making with different preference representation structures: a direct consensus framework and its properties, Knowl. Based Syst. 58 (2014) 45-57 .

7. Z. P. Fan and Y. Zhang, A goal programming approach to group decision-making with three formats of incomplete preference relations, Soft Comput. 14 (10) (2010) 1083-1090.

8. Z. W. Gong, Least-square method to priority of the fuzzy preference relations with incomplete information, Int. J. Approx. Reason. 47 (2) (2008) 258-264.

9. E. Herrera-Viedma, F. Herrera and F. Chiclana, A consensus model for multiperson decision making with different preference structures, IEEE Transactions on Systems, Man, and Cybernetics Part-A: Systems and Humans /bf 32 (3) (2002) 394-402.

10. E. Herrera-Viedma, F. Herrera, F. Chiclana and M. Luque, Some issues on consistency of fuzzy preference relations, Eur. J. Oper. Res. 154 (1) (2004) 98109.

11. E. Herrera-Viedma, F. Chiclana, F. Herrera and S. Alonso, Group decision-making model with incomplete fuzzy preference relations based on additive consistency, IEEE Transactions on Systems, Man and Cybernetics Part-B: Cybernetics 37 (1) (2007) 176-189.

12. E. Herrera-Viedma, S. Alonso, F. Chiclana and F. Herrera, A consensus model for group decision making with incomplete fuzzy preference relations, IEEE Transactions on Fuzzy Syst. 15 (5) (2007) 863-877.

13. E. Herrera-Viedma , F. J. Cabrerizo , J. Kacprzyk and W. Pedrycz, A review of soft consensus models in a fuzzy environment, Inf. Fusion. 17 (2014) 4-13.

14. X. Li and X. Chen, Multi-criteria group decision making based on trapezoidal intuitionistic fuzzy information, Appl. Soft Comput. 30 (2015) 454-461.

15. T. Tanino, Fuzzy preference orderings in group decision making, Fuzzy Sets Syst. 12 (2) (1984) 117-131.

16. R. Ureña , F. Chiclana , H. Fujita and E. HerreraViedma , Confidence-consistency driven group decision making approach with incomplete reciprocal intuitionistic preference relations, Knowl. Based Syst. 89 (2015) 86-96.

17. Z. J. Wang and K. W. Li , A multi-step goal programming approach for group decision making with incomplete interval additive reciprocal comparison matrices, Eur. J. Oper. Res. 242 (3) (2015) 890-900.

18. J. Wu and F. Chiclana, Multiplicative consistency of intuitionistic reciprocal preference relations and its application to missing values estimation and consensus building, Knowledge Based Syst. 71 (2014) 187200.

19. J. Wu , F. Chiclana and E. Herrera-Viedma , Trust based consensus model for social network in an incomplete linguistic information context, Appl. Soft Comput. 35 (2015) 827-839. 
20. Y. Xu, R. Patnayakuni and H. Wang, Logarithmic least squares method to priority for group decision making with incomplete fuzzy preference relations, Appl. Math. Modell. 37 (4) (2013) 2139-2152.

21. Y. Xu and H. Wang, Eigenvector method, consistency test and inconsistency repairing for an incomplete fuzzy preference relation, Appl. Math. Modell. 37 (7) (2013) 5171-5183.

22. Y. Xu and H. Wang, A least deviation method for priority derivation in group decision making with incomplete reciprocal preference relations, Int. J. Approx. Reason. 66 (2015) 91-102.

23. Y. Xu, F. J. Cabrerizo and E. Herrera, A consensus model for hesitant fuzzy preference relations and its application in water allocation management, Appl.
Soft Comput. 65 (2017) 265-284.

24. L. A. Zadeh, Fuzzy sets, Inform. Control. 8 (3) (1965) 338-353.

25. X. Zhang, B. Ge, J. Jiang and Y. Tan, Consensus building in group decision making based on multiplicative consistency with incomplete reciprocal preference relations, Knowledge Based Syst. 106 (2016) 96-104.

26. X. Zhang, B. Ge , J. Jiang and K. Yang, A new consensus model for group decision making using fuzzy linguistic preference relations with heterogeneous experts, J. Intell. Fuzzy Syst. 30 (1) (2016) 171-182 .

27. X. Zhang, B. Ge, Y. Tan, A consensus model for group decision making un- der interval type-2 fuzzy environment, Front. Inf. Technol. Electron. Eng. 17 (3) (2016) 237-249 . 